\title{
Avaliação da terapia com fluido no período perioperatório da ovariossalpingohisterectomia, em cadelas com piometra e insuficiência renal aguda
}

\section{Avaliation of perioperative conservative treatment in pyometra ovariohysterectomy bitches of in acute renal failure}

\author{
Paulo Cesar de Carvalho FERREIRA ${ }^{1}$; Angelo João STOPIGLIA ${ }^{2}$; Clair Motos de OLIVEIRA ${ }^{2}$; Lucia \\ da Conceição ANDRADE ${ }^{3}$; Denise Tabachi FANTONI'² Dulce BARBOSA ${ }^{4}$; Marcelo FAUSTINO²; \\ Mariana Semião Francisco TALIB ${ }^{2}$; Maria Heloisa Massola SCHINIZU ${ }^{3}$
}

\author{
${ }^{1}$ Divisão de Assistência Veterinária de Jockey Club de São Paulo, São Paulo-SP e Faculdade de Medicina Veterinária da \\ Universidade do Grande ABC, Santo André-SP \\ ${ }^{2}$ Faculdade de Medicina Veterinária e Zootecnia da Universidade de São Paulo, São Paulo- SP \\ ${ }^{3}$ Faculdade de Medicina da Universidade de São Paulo, São Paulo- SP \\ ${ }^{4}$ Faculdade de Enfermagem da Universidade Federal de São Paulo, São Paulo- SP
}

\begin{abstract}
Resumo
A insuficiência renal aguda foi avaliada em 351 cadelas com piometra, selecionando-se 132 pelos critérios: creatinina $\geq 2,4$ $\mathrm{mg} / \mathrm{dL}$ e/ou ureia $\geq 80 \mathrm{mg} / \mathrm{dL}$ e/ou aumentos relativos de $100 \%$ de creatinina e/ou ureia séricas, no diagnóstico, 24 horas após a ovariossalpingohisterectomia e na retirada dos pontos. Foi administrado Ringer lactato de sódio até $90 \mathrm{~mL} / \mathrm{kg} / \mathrm{h}$, durante quatro horas, ou três dias. Os parâmetros avaliados antes e após cada terapia foram: peso, pressão arterial sistólica (PAS), frequência cardíaca, função renal, hemogasimetria arterial, bioquímica sérica e urinária, unidade de remoção de ureia, fração de excreção de eletrólitos e clearance de creatinina. A análise univariada detectou diferença significante $(\mathrm{p}<0,05)$ para: PAS, pH urinário, peso corpóreo, ureia e creatinina séricas, fração de excreção de eletrólitos e clearance da creatinina. Comparadas as sobreviventes $(79,6 \%)$ com as não sobreviventes $(20,4 \%)$, respectivamente, detectou-se creatinina sérica de $2,38 \pm 1,33$ $\mathrm{mg} / \mathrm{dL}$ (média \pm desvio padrão) e mediana $2,2 \mathrm{mg} / \mathrm{dL}$ e de $5,54 \pm 3,0 \mathrm{mg} / \mathrm{dL}$ (média \pm desvio padrão) e mediana $4,4 \mathrm{mg} / \mathrm{dl}$, com diferença significante $(\mathrm{p}<0,0001)$. Quanto ao clearance da creatinina endógena nas sobreviventes, $1,82 \pm 1,74 \mathrm{~mL} / \mathrm{kg} /$ min (média \pm desvio padrão) e mediana $1,34 \mathrm{mg} / \mathrm{dL}$ e as não sobreviventes $0,36 \pm 0,38 \mathrm{mg} / \mathrm{dL}$ (média \pm desvio padrão) e mediana $0,23 \mathrm{mg} / \mathrm{dL}$, com diferença significativa $(\mathrm{p}<0,0001)$. Conclui-se que o critério de inclusão pode auxiliar na seleção dos animais em estado grave e o clearance da creatinina, considerado relevante quando observados valores $\geq 1 \mathrm{~mL} / \mathrm{kg} / \mathrm{min}$; negativo com valores $<1 \mathrm{~mL} / \mathrm{kg} / \mathrm{min}$ e indicador de falência renal grave e ou morte para valores $\leq 0,5 \mathrm{ml} / \mathrm{kg} / \mathrm{min}$.
\end{abstract}

Palavras-chave: Fluidoterapia. Clearance. Rins. Cães. Equilíbrio ácido-base. Piometra.

\begin{abstract}
Acute renal failure (ARF) was evaluated prospectively in 351 female dogs with piometra, 132 animals were selected for this study, fulfilling the following criteria, measured in three moments (immediately after the diagnosis, 24 hours after the hysterectomy and when the suture was removed): creatinine $\geq 2.4 \mathrm{mg} / \mathrm{dL}$ and/or urea $\geq 80 \mathrm{mg} / \mathrm{dL}$ and/or relative increases of $100 \%$ in comparison with values previously obtained. The animals selected for the prospective study received conservative treatment consisting in lactated Ringer solution, with the maximum dose of $90 \mathrm{~mL} / \mathrm{kg} / \mathrm{hr}$, during four hours. The factors evaluated before and after the therapy were: weight, systolic arterial pressure, heart rate, renal function, arterial hemogasometry, serum and urinary biochemistry and the endogenous renal creatinine clearance. The evaluated parameters showed significant statistical differences ( $\mathrm{p}<0.05$ ) when companing: PAS (systolic arterial pressure), urinary $\mathrm{pH}$, wheigt, serum urea and creatinine, excretion electrolyte rate and creatinine clearance. Howerer, the survining animals (79.6\%) that received the treatment described above showed decresead levels of serum creatinine- $2,38 \pm 1,33 \mathrm{mg} / \mathrm{dL}$ and median $2.2 \mathrm{mg} / \mathrm{dL}$ - when compared to the non-survining animals $(20.4 \%)$ that received the same treatment $-5.54 \pm 3.0 \mathrm{mg} / \mathrm{dL}$ and median $4.4 \mathrm{mg} / \mathrm{dL}-(\mathrm{p}<0.0001)$. When comparing the endogenous creatinine clearance, the survining animals had higher values $-1.82 \pm 1.74 \mathrm{~mL} / \mathrm{kg} / \mathrm{min}$ and median $1.34 \mathrm{~mL} / \mathrm{kg} /$ $\mathrm{min}$ - than the non-survining animals $-0.36 \pm 0.38 \mathrm{~mL} / \mathrm{kg} / \mathrm{min}$ and median $0.23 \mathrm{~mL} / \mathrm{kg} / \mathrm{min}-(\mathrm{p}<0.0001)$. Concluding: the criteria used to include the animals in this study can help to select the severely affected animals. Values higher than $1 \mathrm{~mL} / \mathrm{kg} / \mathrm{min}$ are considered as a good prognosis while values lower than $1 \mathrm{~mL} / \mathrm{kg} / \mathrm{min}$ are considered as a restricted prognosis. Values lower than $0.5 \mathrm{~mL} / \mathrm{kg} / \mathrm{min}$ are considered as strong predictors of severe renal failure or death.
\end{abstract}

Keywords: Fluid terapy. Clearance. Kidney. Dogs. Acid-base status. Piometra.

Correspondência para:

Paulo Cesar de Carvalho Ferreira

Rua da Bica, 410, Ap 154 Bloco C, Freguesia do Ó, CEP 02925000, São Paulo-SP

e-mails: pauloferreira@uniabc.brou admobras@jockeysp.com.br.
Recebido: 10/07/2007

Aprovado: 01/04/2010 


\section{Introdução}

A piometra é uma síndrome que acomete as cadelas, causando uma variedade de manifestações clínicas e laboratoriais com lesões no trato genital e outros órgãos ${ }^{1}$. Alterações bioquímicas tais como hiperproteinemia, hiperglobulinemia, azotemia, proteinúria ou isosteinúria podem ser notadas em um terço das cadelas com a afecção ${ }^{2}$. Quanto às alterações renais, estas parecem ocorrer secundariamente à glomerulonefrite causada por deposição de imunocomplexos e endotoxinas bacterianas, que alteram a resposta das células tubulares dos néfrons ao hormônio antidiurético $(\mathrm{ADH})^{2}$. A insuficiência renal aguda (IRA) que ocorre na piometra pode ser considerada de grau moderado segundo Berren$\operatorname{sen}^{3}$, pois neste estudo foram investigados os desequilíbrios hidreletrolítico e ácido-básico em 119 animais, em que foram detectadas alcalose respiratória e compensação metabólica atribuída à hiperventilação, achados frequentes também nos casos de septicemia ${ }^{4}$. Ainda em relação à gravidade da IRA, observou-se que não havia relação entre a intensidade da azotemia e a taxa de sobrevivência, sugerindo que o prognóstico e a conduta terapêutica não deveriam ser baseados simplesmente nos valores séricos de creatinina e ureia ${ }^{5}$.

Stone et al. ${ }^{6}$ descreveram que $75 \%$ das cadelas com piometra e não azotêmicas apresentavam diminuição da filtração glomerular, em relação aos valores de referência, $2,08 \pm 0,52 \mathrm{~mL} / \mathrm{kg} / \mathrm{min}$ de clearance de creatinina endógena, um método mais objetivo para avaliação da filtração glomerular. A fluidoterapia é muito importante, pois, na azotemia pré-renal, observa-se diminuição considerável nos valores de ureia, creatinina e fósforo séricos, após o tratamento. Já os animais com azotemia que não respondem à fluidoterapia, apresentam prognóstico reservado e possivelmente insuficiência renal primária instalada ${ }^{7}$, observada após o procedimento de ovariossalpin- gohisterectomia $(\mathrm{OSH})$, em 17\% das 58 cadelas com piometra avaliadas por Stone et al. ${ }^{6}$.

Sendo assim, a fluidoterapia é essencial para estabilizar a hemodinâmica e o equilíbrio ácido-básico dos animais desidratados ou em síndrome da resposta inflamatória sistêmica (SIRS) antes da intervenção cirúrgia ${ }^{7}$. A solução de Ringer lactato de sódio é a mais utilizada $^{8}$ para adequação da reposição volêmica na piometra; Mueller e Ludwig ${ }^{9}$ mensuravam a pressão arterial sistólica (PAS) durante a reposição volêmica e valores menores que $90 \mathrm{mmHg}$ eram considerados como hipotensão 9 . Há poucos estudos que avaliam se o animal é responsivo ou não a fluidoterapia. Constam como resposta positiva: pressão arterial superior a $90 \mathrm{mmHg}$, aumento do débito urinário, diminuição considerável da azotemia ${ }^{10}$, parâmetros que são por demais inespecíficos, visto não estabelecer índices para as respostas positivas e negativas, o que acaba por postergar outras terapias ou procedimentos diagnósticos, refletindo assim em incremento na morbidade e mortalidade.

Sendo assim, o objetivo deste trabalho foi avaliar o tratamento com fluido nestes animais, elaborando-se critério de adequação que possibilite, após cada sessão de fluidoterapia, determinar de maneira objetiva se o animal tratado foi responsivo ou não à terapia instituída.

\section{Material e Método}

Foram utilizadas 351 cadelas com piometra, diagnosticadas por exame físico e/ou ultrassonográfico, além da avaliação laboratorial (hemograma, perfil bioquímico sérico e exame de urina), no período de 22 de outubro de 2004 a 17 de fevereiro de $2006^{11}$. Foram então reavaliadas decorridas 24 horas da realização da OSH e no momento da retirada de sutura cutânea, com a realização de novo exame físico, determinação sérica de ureia e creatinina e exame de urina. De posse destes dados, 132 animais foram selecionados confor- 
me o critério de inclusão: creatinina $\geq 2,4 \mathrm{mg} / \mathrm{dL}$ e/ou ureia $\geq 80 \mathrm{mg} / \mathrm{dL}$ e/ou aumentos relativos de $100 \%$ de creatinina e/ou ureia séricas: no diagnóstico, 24 horas após a ovariossalpingohisterectomia e na retirada dos pontos (Figura 1). Os animais selecionados foram submetidos à avaliação de parâmetros hemodinâmicos, metabólicos e respiratórios, antes e após cada sessão de fluidoterapia. A veia cefálica foi cateterizada, para receber fluidoterapia com solução de Ringer com lactato, na dose de até $90 \mathrm{~mL} / \mathrm{kg} / \mathrm{hora}^{9}$, durante quatro horas e por três dias consecutivos ${ }^{10}$, baseada nos achados dos exames físico e laboratoriais. O volume (mililitros) utilizado, foi baseado na seguinte forma: Volume $(\mathrm{mL})=$ peso $(\mathrm{kg}) \times$ \% desidratação; o grau de desidratação, discreta (4\%), moderada (6 a $8 \%$ ) e intensa $(10 \text { a } 12 \%)^{12}$. O volume obtido, $10 \%$ era administrado em um período de 20 minutos, mediante mensuração da frequência cardíaca, auscultação car- díaca e pulmonar, tempo de perfusão capilar, coloração das mucosas, débito urinário $(\mathrm{ml} / \mathrm{kg} / \mathrm{h})$ e pressão arterial sistólica $(\mathrm{mmHg})$ e os valores inferiores à $90 \mathrm{mmHg}$, caracterizaram hipotensão .

A bexiga urinária era esvaziada, uma amostra colhida e encaminhada para análise laboratorial (Combur Teste ${ }^{\circledR}$ e sedimentoscopia) e o débito urinário era monitorado para diferenciar a presença de oligúria $(<1 \mathrm{~mL} / \mathrm{kg} / \mathrm{h})$, poliúria $(>2 \mathrm{~mL} / \mathrm{kg} / \mathrm{h})$, anúria $(0 \mathrm{~mL} /$ $\mathrm{kg} / \mathrm{h}$ ) ou diurese normal $(1-2 \mathrm{~mL} / \mathrm{kg} / \mathrm{h})^{10}$. A bioquímica urinária foi avaliada por meio de mensuração do sódio, potássio, ureia e creatinina.

A hemodinâmica central foi mensurada por auscultação dos batimentos cardíacos, a cada 15 minutos; a PAS monitorada de forma não invasiva, a cada cinco minutos, pelo método oscilométrico. Foram considerados valores normais: PAS entre 110 a $160 \mathrm{mmHg}^{13}$. A avaliação da oxigenação, da ventilação e do equilí-

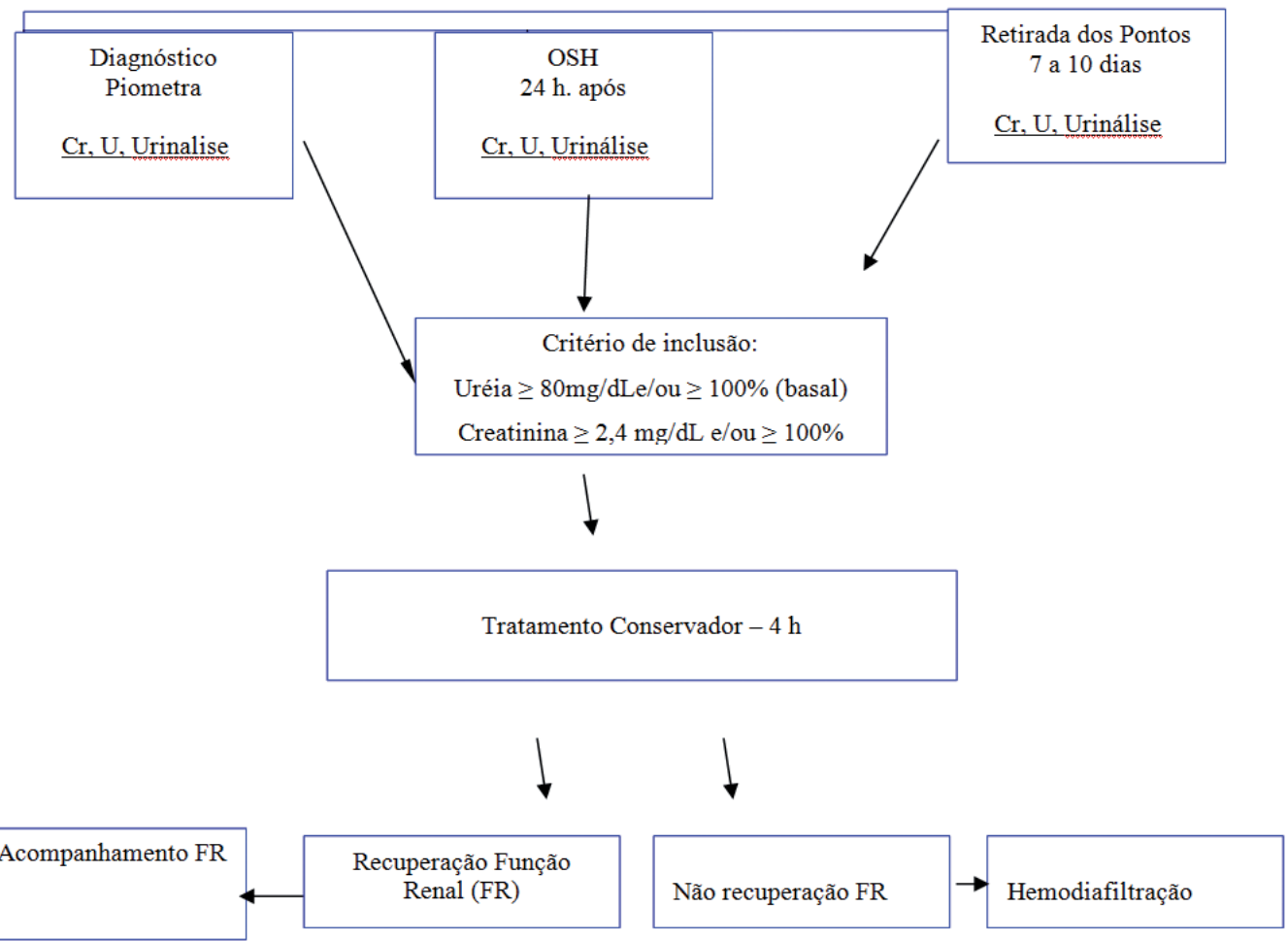

Figura 1 - Critério de inclusão utilizado no estudo da avaliação do tratamento com fluido no período perioperatório da ovariossalpingohisterectomia, em cadelas com piometra e insuficiência renal aguda [Creatinina sérica (Cr), Ureia sérica (U), Ovariossalpingohisterectomia (OSH) e Função renal (FR)] 
brio ácido-básico foi realizada pela colheita de sangue arterial por punção percutânea da artéria femural e as determinações realizadas em analisador de gases sanguíneos e pH. Foram avaliadas as concentrações séricas de $\mathrm{Na}^{+}, \mathrm{K}^{+}$e $\mathrm{Cl}^{-}$.

O perfil bioquímico sérico renal foi avaliado pela determinação da ureia e creatinina e também foram mensurados outros índices urinários, o clearance da creatinina endógena e as frações de excreção do sódio, potássio e cloreto. O clearance da creatinina (Ccr) ( $\mathrm{mL} / \mathrm{kg} / \mathrm{min})$, foi calculado pela fórmula:

$\mathrm{Ccr}=\mathrm{Cru}(\mathrm{mg} / \mathrm{dL}) \times \mathrm{Vu}(\mathrm{mL}) \div \mathrm{Cs}(\mathrm{mg} / \mathrm{dL}) \times$ Peso (kg) x Tempo (min), onde Cru, a creatinina urinária e Cs, a creatinina sérica, valor de normalidade: 2,15 \pm $0,09 \mathrm{~mL} / \mathrm{kg} / \mathrm{min}^{14}$. A fração de excreção dos eletrólitos (FE): FEx $(\%)=\mathrm{Eu}(\mathrm{mmol} / \mathrm{L}) \times \mathrm{Cs}(\mathrm{mg} / \mathrm{dL}) \div \mathrm{Es}$ $(\mathrm{mmol} / \mathrm{L}) \times \mathrm{Cru}(\mathrm{mg} / \mathrm{dL}) \times 100$, onde Eu, o eletrólito na urina; o Es, o eletrólito no soro.

Para a adequação do tratamento com fluido foi utilizado também o índice de remoção de ureia (URR): URR $=$ [ureia final $]-[$ ureia inicial $] \div$ [ureia inicial $]$ mediante sua mensuração antes e após cada tratamento realizado ${ }^{15}$. Considerou-se adequado URR, na IRA de $25-33 \%$, no $1^{\circ}, 2^{\circ}$ e $3^{\circ}$ dias $^{16}$.

Os resultados foram apresentados como frequência em porcentagem e/ou média \pm desvios-padrão. As variáveis foram tratadas pelo teste de normalidade e utililizados testes paramétricos. Para comparação entre variáveis numéricas foi utilizado o "teste t de student" não pareado ou pareado de acordo com os dados. Entre variáveis categóricas, foi utilizado o teste qui-quadrado.

Para a análise univariada da frequência cardíaca, pressão arterial sistólica, peso, hemogasometria arterial e eletrólitos, ureia e creatinina séricos, clearance da creatinina endógena, fração de excreção de eletrólitos, parâmetros hematológicos, débito urinário, dose de fluido, índice de remoção de ureia (URR), exame de urina tipoI; eletrólitos, ureia e creatinina urinários, o programa estatístico utilizado para todos os cálculos foi o GraphPad Prism Version 3.0, 1999 (GraphPad Prism [homepage on the internet]. GraphPad Prism Version 3.0, 1999 [cited 2006 Jan 26]. Available from: http://www.graphpad.com/prism/Prism.htm).

\section{Resultados}

Das 132 cadelas, 81 foram selecionadas no momento do diagnóstico; 39 cadelas 24 horas após a OSH e apenas 12 na remoção da sutura cutânea. No momento do diagnóstico $(\mathrm{n}=81), 47$ cadelas necessitaram de apenas uma sessão de fluido, 29 utilizaram duas sessões e cinco necessitaram de três sessões de fluido. Quanto às cadelas selecionadas 24 horas após a OSH $(\mathrm{n}=39)$, 12 receberam uma sessão de fluido; 20 necessitaram de duas sessões e uma delas, frente à gravidade do caso, recebeu hemodiafiltração e sobreviveu, e sete cadelas foram submetidas a três sessões de tratamento com fluido, devido à necessidade do quadro clínico. Em relação às outras 12 selecionadas no momento da remoção da sutura cutânea, seis cadelas receberam uma sessão de fluido, quatro receberam duas sessões e outras duas necessitaram de três sessões.

Entre as cadelas selecionadas que receberam o tratamento com fluido, 14 morreram em diferentes momentos: 11 (11/14) morreram (selecionadas no momento do diagnóstico), sendo que seis receberam apenas uma sessão de fluido; três receberam duas sessões de fluido (uma delas apresentou pneumonia e foi eutanasiada) e as outras duas receberam três sessões de fluido, porém, frente à gravidade do quadro clínico, foram tratadas com a hemodiafiltração (HDF); no entanto uma delas morreu durante a sessão de HDF e a outra sobreviveu por sete dias. Já das outras três (3/14) cadelas que morreram (selecionadas 24 horas após a OSH), uma recebeu apenas uma sessão de fluido e as outras duas receberam duas sessões de fluido; frente à gravidade do quadro clínico destas duas cadelas não responsivas ao tratamento com fluido, foi realizada a HDF, uma cadela morreu durante a sessão 
e a outra cadela sobreviveu 21 dias após as três sessões de HDF.

Os parâmetros avaliados em relação aos momentos antes e após cada sessão de fluido apresentaram os seguintes resultados: em relação à hemodinâmica central não foram observadas diferenças na frequência cardíaca, mas a PAS apresentou aumento $(\mathrm{p}<0,001)$ (Tabela 1). Quanto à avaliação do peso corpóreo houve uma diminuição no segundo dia do tratamento, em relação ao primeiro dia após o tratamento $(\mathrm{p}<$ 0,05) (Tabela 1).

Já a hemogasometria arterial e os eletrólitos séricos não apresentaram alteração quando mensurados. No entanto o sódio sérico e o excesso de bases, quando observados os seus valores médios e desvio padrão, apresentavam uma tendência à elevação, porém não eram significativos (Tabela 2).

A função renal quando analisada pela mensuração da ureia e creatinina séricas e clearance da creatinina endógena, fração de excreção dos eletrólitos sódio, potássio e cloreto, todas as variáveis apresentavam diferença após o tratamento (Tabela 2).

Quanto aos parâmetros hematológicos avaliados, o número de hemácias e leucócitos não se alterou, ocorrendo apenas neutropenia $(\mathrm{p}<0,05)$ (Tabela 3$)$.

Em relação ao $1^{\circ}, 2^{\circ}$ e $3^{\circ}$ dia de tratamento com fluído, não houve diferença em relação ao débito urinário, dose do fluído e URR (Tabela 4), mas a URR nos animais submetidos à HDF foi significante ( $\mathrm{p}<$ 0,05). Em relação aos parâmetros urinários foi possí- vel observar que o $\mathrm{pH}$ era menor no primeiro dia de tratamento $(\mathrm{p}<0,05)$, aumentando durante o segundo e terceiro dias $(\mathrm{p}<0,05)$, já a densidade urinária não se alterou (Tabela 5). Quanto aos outros parâmetros urinários, não foram observadas diferenças (Tabela 5). Em relação à avaliação da bioquímica urinária não foi possível detectar alteração significante $(\mathrm{p}>0,05)$ nas concentrações de sódio, potássio e cloreto, e o mesmo ocorreu com a ureia e creatinina urinária, quando eram analisados isoladamente e não considerando o clearance da creatinina (Tabela 5). Observou-se que as cadelas sobreviventes apresentaram Ccr de $1,82 \pm 1,74 \mathrm{~mL} / \mathrm{kg} / \mathrm{min}$ (média \pm desvio padrão) e mediana $1,34 \mathrm{~mL} / \mathrm{kg} / \mathrm{min}$ e para as não sobreviventes os valores de Ccr foram 0,36 \pm $0,38 \mathrm{~mL} / \mathrm{kg} / \mathrm{min}$ (média \pm desvio padrão) e mediana $0,23 \mathrm{~mL} / \mathrm{kg} / \mathrm{min}$ com diferença significante $(\mathrm{p}<$ 0,0001).

\section{Discussão}

Os animais submetidos ao tratamento foram responsivos ou não à terapia com fluido? Sim, observouse que em $79,6 \%$ dos animais tratados recuperavam a função renal ${ }^{9} e$ o período de quatro horas de fluidoterapia foram suficientes para responder a pergunta, pois foram responsivos ${ }^{10}$, mediante avaliação do exame físico e dos parâmetros laboratoriais, principalmente pelo clearance da creatinina $(>1 \mathrm{~mL} / \mathrm{kg} /$ min). Quando não responsivo, o animal deveria ser submetido à hemodiafiltração, estratégia para dimi-

Tabela 1 - Valores médios (VM) e seus respectivos desvios padrão (DP) da frequência cardíaca (FC), pressão arterial sistólica (PAS) e peso corpóreo, antes e depois do tratamento com fluido, nos dias 1,2 e 3 de cadelas com piometra ( $n=132)$

\begin{tabular}{|c|c|c|c|c|c|c|}
\hline & \multicolumn{2}{|c|}{$1^{\circ}$ dia $\quad \mathrm{N}=81$} & \multicolumn{2}{|c|}{$2^{\circ}$ dia $\quad \mathrm{N}=39$} & \multicolumn{2}{|c|}{$3^{\circ}$ dia $\mathrm{N}=12$} \\
\hline & antes & depois & antes & depois & antes & depois \\
\hline $\begin{array}{l}\mathrm{FC} \\
\mathrm{Bat} / \mathrm{min}\end{array}$ & $123 \pm 29$ & $114 \pm 16$ & $134 \pm 19,8$ & $123 \pm 14$ & $129 \pm 13$ & $107 \pm 13$ \\
\hline $\begin{array}{l}\mathrm{PAS}^{* *} \\
\mathrm{mmHg}\end{array}$ & $104 \pm 14$ & $136 \pm 10$ & $113 \pm 14$ & $126 \pm 18$ & $119 \pm 22$ & $136 \pm 20$ \\
\hline $\begin{array}{l}\mathrm{Peso}^{*} \\
\mathrm{Kg} \\
\end{array}$ & $17,7 \pm 10,97$ & $18,2 \pm 11,6^{*}$ & $15,82 \pm 11,08^{*}$ & $17,18 \pm 11,5^{\star}$ & $15,61 \pm 11,32$ & $16,62 \pm 11,35$ \\
\hline
\end{tabular}

${ }^{*} \mathrm{p}<0,05 ;{ }^{* *} \mathrm{p}<0,0001$ 
Tabela 2 - Valores médios (MD) e seus respectivos desvios padrão (DP) dos parâmetros da hemogasimetria arterial, eletrólitos, ureia e creatinina séricos, $\mathrm{FE}_{\mathrm{Na}+}, \mathrm{FE}_{\mathrm{K}+}, \mathrm{FE}_{\mathrm{CL}-}$ e o $\mathrm{C}_{\mathrm{cr}}$, antes e depois do tratamento com fluido, nos dias 1,2 e 3 de cadelas com piometra $(\mathrm{n}=132)$

\begin{tabular}{|c|c|c|c|c|c|c|c|}
\hline & & \multicolumn{2}{|c|}{$1^{\circ}$ dia $N=81$} & \multicolumn{2}{|c|}{$2^{\circ}$ dia $\mathrm{N}=39$} & \multicolumn{2}{|c|}{$3^{\circ}$ dia $\mathrm{N}=12$} \\
\hline & & antes & depois & antes & depois & antes & depois \\
\hline \multirow{2}{*}{$\begin{array}{c}\mathrm{PaO}_{2} \\
\mathrm{~mm} / \mathrm{Hg}\end{array}$} & $\mathrm{MD}$ & 82,9 & 84,6 & 87,2 & 88,7 & 85,0 & 90,1 \\
\hline & $\mathrm{DP}$ & 8,49 & 11,0 & 8,6 & 9,1 & 10,9 & 8,7 \\
\hline \multirow{2}{*}{$\begin{array}{c}\mathrm{SaO}_{2} \\
\mathrm{mmHg}\end{array}$} & $\mathrm{MD}$ & 92,60 & 94,7 & 93,0 & 94,5 & 92,14 & 93,4 \\
\hline & $\mathrm{DP}$ & 5,32 & 2,77 & 3,80 & 3,02 & 3,93 & 2,81 \\
\hline \multirow{2}{*}{$\begin{array}{c}\mathrm{CaO}_{2} \\
\mathrm{~mL} / 1000 \mathrm{~L}\end{array}$} & $\mathrm{MD}$ & 15,43 & 15,41 & 16,74 & 18,0 & 15,14 & 15,5 \\
\hline & $\mathrm{DP}$ & 3,80 & 3,48 & 3,24 & 3,78 & 2,75 & 2,83 \\
\hline \multirow[t]{2}{*}{$\mathrm{pH}$} & $\mathrm{MD}$ & 7,32 & 7,33 & 7,28 & 7,35 & 7,30 & 7,37 \\
\hline & $\mathrm{DP}$ & 0,1 & 0,06 & 0,12 & 0,07 & 0,16 & 0,06 \\
\hline \multirow{2}{*}{$\begin{array}{l}{ }^{*} \mathrm{PaCO}_{2} \\
\mathrm{~mm} / \mathrm{Hg}\end{array}$} & $\mathrm{MD}$ & 30,9 & 30,7 & 33,43 & 34,3 & 31,5 & 35,16 \\
\hline & $\mathrm{DP}$ & 8,25 & 8,53 & 8,37 & 8,45 & 6,96 & 12,3 \\
\hline \multirow{2}{*}{$\begin{array}{l}{ }^{*} \mathrm{HCO}_{3}^{-} \\
{ }^{\mathrm{Mmol} / \mathrm{L}}\end{array}$} & $\mathrm{MD}$ & 15,63 & 18,63 & 17,87 & 18,48 & 14,8 & 19,85 \\
\hline & $\mathrm{DP}$ & 4,47 & 5,94 & 5,05 & 4,87 & 5,44 & 5,03 \\
\hline \multirow{2}{*}{$\begin{array}{c}\mathrm{BE} \\
\mathrm{Mmol} / \mathrm{L}\end{array}$} & $\mathrm{MD}$ & $-8,7$ & $-6,8$ & $-8,6$ & $-4,9$ & $-8,3$ & $-6,1$ \\
\hline & $\mathrm{DP}$ & 4,83 & 4,57 & 6,81 & 1,63 & 2,49 & 1,86 \\
\hline \multirow{2}{*}{$\begin{array}{c}\mathrm{Na}^{+} \\
\mathrm{Mmol} / \mathrm{L}\end{array}$} & $\mathrm{MD}$ & 144,5 & 147,2 & 146,1 & 148,4 & 152,7 & 153,0 \\
\hline & $\mathrm{DP}$ & 9,9 & 8,7 & 10,8 & 5,7 & 5,9 & 7,9 \\
\hline \multirow{2}{*}{$\begin{array}{c}\mathrm{K}^{+} \\
\mathrm{Mmol} / \mathrm{L}\end{array}$} & $\mathrm{MD}$ & 4,6 & 4,53 & 4,25 & 3,7 & 4,6 & 4,31 \\
\hline & $\mathrm{DP}$ & 1,1 & 1,0 & 1,15 & 0,55 & 1,3 & 1,15 \\
\hline \multirow{2}{*}{$\begin{array}{c}\mathrm{Cl}^{-} \\
\mathrm{Mmol} / \mathrm{L}\end{array}$} & $\mathrm{MD}$ & 112,3 & 114,8 & 114,7 & 114,8 & 116,9 & 118,8 \\
\hline & $\mathrm{DP}$ & 12,68 & 8,48 & 14,8 & 15,0 & 8,73 & 4,21 \\
\hline \multirow{2}{*}{$\begin{array}{l}{ }^{*} \text { Ureia } \\
\mathrm{mg} / \mathrm{dL}\end{array}$} & $\mathrm{MD}$ & 190 & 131 & 84,1 & 71,34 & 53,9 & 49,9 \\
\hline & $\mathrm{DP}$ & 130 & 84,2 & 55,7 & 43,2 & 27 & 29,3 \\
\hline \multirow{2}{*}{$\begin{array}{c}{ }^{*} \mathrm{Creat} \\
\mathrm{mg} / \mathrm{dL} \\
\mathrm{N}=\end{array}$} & $\mathrm{MD}$ & 3,1 & 2,6 & 1,76 & 1,45 & 1,41 & 1,05 \\
\hline & $\mathrm{DP}$ & 2,3 & 2,24 & 1,24 & 1,21 & 1,01 & 0,79 \\
\hline \multirow{2}{*}{$\begin{array}{c}{ }^{*} \mathrm{FE}_{\mathrm{Na}} \\
\%\end{array}$} & $\mathrm{MD}$ & 2,46 & 9,3 & 5,69 & 3,81 & 6,57 & 6,3 \\
\hline & $\mathrm{DP}$ & 5,16 & 12,67 & 10,83 & 3,45 & 12,7 & 3,94 \\
\hline \multirow{2}{*}{$\begin{array}{c}{ }^{*} \mathrm{FE}_{\mathrm{K}+} \\
\%\end{array}$} & $\mathrm{MD}$ & 37 & 35,71 & 29,5 & 16,4 & 21,3 & \\
\hline & $\mathrm{DP}$ & 35,71 & 26,6 & 30 & 14 & 31 & \\
\hline \multirow{2}{*}{${ }_{\%}^{*}{ }_{\%} \mathrm{FE}_{\mathrm{Cl}}$} & $\mathrm{MD}$ & 2,54 & 12,51 & 6,53 & 10,45 & 9,06 & 6,11 \\
\hline & $\mathrm{DP}$ & 6,62 & 16,34 & 12,4 & 15,6 & 16 & 6 \\
\hline \multirow{2}{*}{$\begin{array}{c}{ }^{*} \mathrm{C}_{\mathrm{cr}} \\
\mathrm{ml} / \mathrm{Kg} / \mathrm{min}\end{array}$} & $\mathrm{MD}$ & 1,33 & 1,72 & 2,04 & 2,89 & 2,64 & 3,16 \\
\hline & $\mathrm{DP}$ & 1,59 & 1,88 & 1,36 & 2,8 & 2,12 & 2,09 \\
\hline
\end{tabular}

$\mathrm{PaO}_{2}$ - Pressão Parcial de Oxigênio; $\mathrm{SaO}_{2}$ - Saturação Arterial de Oxigênio; $\mathrm{CaO}_{2}$ - Conteúdo Arterial de Oxigênio; $\mathrm{PaCO}_{2}$ - Pressão Parcial do Dióxido de Carbono; $\mathrm{Cl}^{-}$- Íon Cloreto; $\mathrm{HCO}_{3}^{-}$- Bicarbonato; $\mathrm{BE}$ - Excesso de Bases; $\mathrm{Na}+$ - Íon Sódio; $\mathrm{K}^{+}$- Íon Potássio; $\mathrm{Cl}^{-}$- Íon Cloreto; Creat - Creatinina; $\mathrm{FE}_{\mathrm{Na}}$ - Fração de excreção do íon Sódio; $\mathrm{FE}_{\mathrm{K}+}$ - Fração de excreção do íon Potássio $\mathrm{FE}_{\mathrm{Cl}-}$ - Fração de excreção do íon Cloreto $\mathrm{C}_{\mathrm{cr}}$ - Clearance da Creatinina; ${ }^{*} \mathrm{p}<0,05$

nuir a morbidade e mortalidade nesta doença ${ }^{11,15}$. As principais alterações observadas durante a fluidoterapia foram: êmese, tremores musculares, sialorreia, taquicardia, taquipneia, corrimento nasal e diurese aumentada ${ }^{17}$. Não foi possível utilizar a PAS como parâmetro de adequação, como sugerido pela literatura ${ }^{9}$, pois mesmo os animais que não apresentavam evolução favorável da IRA exibiam PAS compatíveis com a normalidade, após o tratamento (Tabela 1).

Embora a fluidoterapia restabeleça a perfusão dos órgãos inadequadamente perfundidos na endotoxemia da piometra ${ }^{8}$, a sobrecarga de volume pode levar 
Tabela 3 - Valores médios (MD) e seus respectivos desvios padrão (DP) para os parâmetros hematológicos mensurados nos animais antes e depois de submetê-los ao tratamento com fluido, nos dias 1,2 e 3 de cadelas com piometra $(n=132)$

\begin{tabular}{|c|c|c|c|c|c|c|c|}
\hline & & $1^{\circ} \mathrm{dia}$ & $\mathrm{N}=81$ & $2^{\circ} \mathrm{dia}$ & $\mathrm{N}=39$ & $3^{\circ} \mathrm{dia}$ & $\mathrm{N}=12$ \\
\hline & & antes & depois & antes & depois & antes & depois \\
\hline $\mathrm{HE}$ & $\mathrm{MD}$ & 5,6 & 4,6 & 4,4 & 4,5 & 4,54 & 4,0 \\
\hline$\left(\mathrm{x} 10^{6}\right)$ & $\mathrm{DP}$ & 1,68 & 1,56 & 1,4 & 1,29 & 1,26 & 0,97 \\
\hline $\mathrm{Ht}^{*}$ & $\mathrm{MD}$ & 36,0 & 34,0 & 30,9 & 29,2 & 31,2 & 26,5 \\
\hline$\%$ & $\mathrm{DP}$ & 10,2 & 9,8 & 9,7 & 8,0 & 8,1 & 9,1 \\
\hline $\mathrm{Hb}^{*}$ & $\mathrm{MD}$ & 11,8 & 10,75 & 10,3 & 9,3 & 10,2 & 9,4 \\
\hline $\mathrm{g} / \mathrm{dL}$ & $\mathrm{DP}$ & 3,5 & 3,6 & 3,3 & 2,9 & 2,91 & 2,6 \\
\hline $\mathrm{VCM}^{*}$ & MD & 66,3 & 66,0 & 69,5 & 65,2 & 71,2 & 68,2 \\
\hline FL & $\mathrm{DP}$ & 5,7 & 4,48 & 7,88 & 3,14 & 7,37 & 6,13 \\
\hline Leuc & $\mathrm{MD}$ & 32512 & 41637 & 28205 & 35587 & 35266 & 35737 \\
\hline $10^{3} / \mathrm{mm}^{3}$ & $\mathrm{DP}$ & 22682 & 24471 & 14574 & 17822 & 20198 & 9374 \\
\hline Neut $^{*}$ & $\mathrm{MD}$ & 29205 & 40947 & 24394 & 31886 & 17257 & 16927 \\
\hline$/ \mathrm{mm}^{3}$ & $\mathrm{DP}$ & 18691 & 20837 & 16765 & 7719 & 11139 & 13347 \\
\hline Metam & $\mathrm{MD}$ & 1664 & & 1206 & & & \\
\hline$/ \mathrm{mm}^{3}$ & $\mathrm{DP}$ & 1948 & & 997 & & & \\
\hline Bastonetes & $\mathrm{MD}$ & 34000 & 2135 & 1285 & & 3595 & \\
\hline$/ \mathrm{mm}^{3}$ & $\mathrm{DP}$ & 6179 & 3414 & 825 & & 2146 & \\
\hline Eosinóf & MD & 694 & 386 & 458 & & 477 & \\
\hline$/ \mathrm{mm}^{3}$ & $\mathrm{DP}$ & 593 & 391 & 242 & & 211 & \\
\hline Linfócitos & $\mathrm{MD}$ & 2393 & 1292 & 2486 & & 1984 & \\
\hline$/ \mathrm{mm}^{3}$ & $\mathrm{DP}$ & 2227 & 1015 & 2003 & & 1210 & \\
\hline Monócitos & $\mathrm{MD}$ & 3768 & 1948 & 2315 & & 2813 & \\
\hline$/ \mathrm{mm}^{3}$ & $\mathrm{DP}$ & 5030 & 2534 & 2911 & & 2736 & \\
\hline
\end{tabular}

HE - Hemácia; Ht - Hematócrito Hb - Hemoglobina; VCM - Volume Corpuscular Médio; Leuc - Leucócitos; Neut - Neutrófilos; Neut - Neutrófilos; Metam - Metamielócitos; Eosinóf - Eosinófilos; ${ }^{*} \mathrm{p}<0,05$

Tabela 4 - Valores médios (MD) e seus respectivos desvios padrão (DP) do débito urinário, fluido administrado e Unidade de Remoção de Ureia (URR) no tratamento com fluido nos dias 1,2 e 3 de cadelas com piometra $(n=132)$

\begin{tabular}{cccc}
\hline & $1^{\circ}$ dia $\mathrm{N}=81$ & $2^{\circ}$ dia $\mathrm{N}=39$ & $3^{\circ}$ dia $\mathrm{N}=12$ \\
\hline $\begin{array}{c}\text { Débito } \\
\text { Urinário } \\
\mathrm{mL} / \mathrm{kg} / \mathrm{h}\end{array}$ & $3,81 \pm 5,03$ & $3,38 \pm 2,80$ & $2,44 \pm 0,98$ \\
$\begin{array}{c}\text { Fluido } \\
\mathrm{ml} / \mathrm{kg} / \mathrm{h}\end{array}$ & $25,9 \pm 14,54$ & $34,9 \pm 19,14$ & $27,3 \pm 16,12$ \\
$\mathrm{URR}$ & & \\
$\%$ & $22,90 \pm 9,40$ & $20,02 \pm 7,35$ & $17,87 \pm 9,72$ \\
\hline
\end{tabular}

URR - Unidade de Remoção de Ureia

a complicações, como: síndrome de hipertensão intra- $\quad$ fluido ${ }^{12}$; translocações bacterianas ${ }^{18}$ e agravadas tamabdominal, principalmente nos animais submetidos à bém pela imunossupressão na piometra ${ }^{19} \mathrm{o}$ que polaparotomia e administração de grandes volumes de derá precipitar alterações importantes como a falha 
Tabela 5 - Valores médios (VM) na urina do pH, densidade, proteína, hemoglobina, hemácias, leucócitos, cilindros, células epiteliais, sódio, potássio, cloreto, ureia, creatinina e seus respectivos desvios padrão (DP), antes e depois do tratamento com fluido, nos dias 1,2 e 3 de cadelas com piometra $(\mathrm{n}=132)$

\begin{tabular}{|c|c|c|c|c|c|c|c|}
\hline & & \multicolumn{2}{|c|}{$1^{\circ}$ dia $\mathrm{N}=81$} & \multicolumn{2}{|c|}{$2^{\circ}$ dia $\quad \mathrm{N}=39$} & \multicolumn{2}{|c|}{$3^{\circ}$ dia $\mathrm{N}=12$} \\
\hline & & antes & depois & antes & depois & antes & depois \\
\hline \multirow[t]{2}{*}{$\mathrm{PH}^{*}$} & MD & 6,13 & 5,97 & 5,96 & 6,04 & 6,2 & 6,8 \\
\hline & DP & 1,12 & 0,93 & 1,08 & 1,0 & 0,96 & 1,21 \\
\hline \multirow[t]{2}{*}{ Dens } & MD & 1024 & 1020,3 & 1026 & 1020 & 1021 & 1019 \\
\hline & DP & 9,7 & 6,93 & 13,51 & 9,5 & 8,47 & 6,94 \\
\hline \multirow[t]{2}{*}{ Proteína } & $\mathrm{MD}$ & 1,1 & 1,04 & 1,04 & 1,05 & 0,76 & 0,82 \\
\hline & DP & 0,47 & 0,21 & 0,2 & 0,23 & 0,44 & 0,39 \\
\hline \multirow[t]{2}{*}{$\mathrm{Hb}$} & $\mathrm{MD}$ & 2,2 & 5,2 & 2,3 & 2,3 & 1,0 & 0,67 \\
\hline & DP & 1,0 & 6,5 & 1,1 & 1,15 & 0,6 & 0,65 \\
\hline $\mathrm{He}$ & $\mathrm{MD}$ & 4,8 & 5,5 & 2,9 & 4,1 & 3,15 & 3,43 \\
\hline $\mathrm{X} 400$ & DP & 5,7 & 5,8 & 4,5 & 4,5 & 4,61 & 3,11 \\
\hline Leuc & $\mathrm{MD}$ & 3,9 & 3,1 & 2,7 & 3,3 & 2,42 & 4,0 \\
\hline $\mathrm{X} 400$ & DP & 4,1 & 4,0 & 2,8 & 3,0 & 2,8 & 2,7 \\
\hline Cilind & MD & 1,1 & 1,0 & 0,9 & 1,25 & 1,0 & 0,7 \\
\hline $\mathrm{X} 400$ & DP & 0,34 & 0,5 & 0,3 & 0,5 & 0,1 & 0,57 \\
\hline Células Ep & MD & 1,29 & 0,8 & 1,1 & 1,14 & 1,0 & \\
\hline $\mathrm{X} 400$ & DP & 0,6 & 0,44 & 0,73 & 0,37 & 0,0 & \\
\hline $\mathrm{Na}^{+}$ & $\mathrm{MD}$ & 65,02 & 82,05 & 73,03 & 34,6 & 82,1 & 117,3 \\
\hline $\mathrm{mmol} / \mathrm{L}$ & DP & 61,09 & 59,12 & 55,52 & 19,71 & 36,81 & 100,94 \\
\hline $\mathrm{K}^{+}$ & $\mathrm{MD}$ & 37,1 & 27,7 & 36,03 & 23,96 & 22,24 & 19,3 \\
\hline $\mathrm{mmol} / \mathrm{L}$ & DP & 27,3 & 22,83 & 50,3 & 16,4 & 15.4 & 8,63 \\
\hline $\mathrm{Cl}^{-}$ & $\mathrm{MD}$ & 56,24 & 61,75 & 75,65 & 44,7 & 73,0 & 94,6 \\
\hline $\mathrm{mmol} / \mathrm{L}$ & DP & 46,8 & 41,12 & 63,43 & 25,8 & 35,7 & 65,3 \\
\hline Ureia & $\mathrm{MD}$ & 1054 & 795 & 1081,1 & 566,1 & 1456,1 & 724 \\
\hline $\mathrm{mg} / \mathrm{dl}$ & DP & 795,9 & 548,6 & 914,1 & 911,4 & 1386,1 & 1021 \\
\hline Creatinina & $\mathrm{MD}$ & 65,23 & 41,1 & 79,04 & 48,4 & 80,54 & 47,3 \\
\hline $\mathrm{mg} / \mathrm{dl}$ & DP & 48,94 & 28,6 & 66,3 & 52,96 & 43,27 & 23,47 \\
\hline
\end{tabular}

He - Hemácias; Dens - Densidade;Hb - Hemoglobina; Leuc - Leucócitos; Cilind - Cilindros; Células Ep. - Células Epiteliais; $\mathrm{Na}^{+}$Íon Sódio; $\mathrm{K}^{+}$- Íon Potássio; $\mathrm{Cl}^{-}$- Íon Cloreto; ${ }^{*} \mathrm{p}<0,05$

de múltiplos órgãos ${ }^{20}$. Foi encontrado um animal que, recuperado da IRA, apresentou pneumonia grave não remissível às terapias utilizadas e posteriormente $o$ proprietário optou pela eutanásia.

O equilíbrio hidreletrolítico e ácido-básico (Tabela 2) não apresentaram alterações quanto à concentração do íon bicarbonato, $\mathrm{pH}$ arterial e eletrólitos séricos, mas quando observados os valores médios do bicarbonato durante os tratamentos, houve sempre tendência ao aumento. A doença piometra foi estra- tificada ${ }^{3}$ utilizando-se o bicarbonato como referência e concentrações inferiores a $15 \mathrm{mmol} / \mathrm{L}$ eram consideradas graves e coincidiam com os animais que vieram a óbito. Nos animais avaliados neste estudo obtiveram-se concentrações inferiores a $14 \mathrm{mmol} / \mathrm{L}$ nas cadelas que não sobreviveram ${ }^{11}$. Em relação aos valores de $\mathrm{pH}$ e bicarbonato, assumiam valores próximos da normalidade, alterações estas bem documentadas como a alcalose respiratória com compensação metabólica ${ }^{17}$. 
Quantos aos eletrólitos séricos (Tabela 2) a literatura aponta a hipernatremia e hipercloremia como alterações frequentes atribuídas à desidratação ${ }^{7}$. Já nos animais avaliados, no momento do diagnóstico detectouse que 22 animais apresentavam hiponatrêmicos $\left(\mathrm{Na}^{+}<\right.$ $140 \mathrm{mmol} / \mathrm{L})^{17}(22 / 132)$ e cinco animais hipernatrêmi$\cos \left(\mathrm{Na}^{+}>155 \mathrm{mmol} / \mathrm{L}\right)^{17}(05 / 132)$. Os animais hiponatrêmicos com piometra possivelmente se apresentavam com diminuição da osmolaridade sanguínea, além de hipovolêmicos, quadro este grave ${ }^{17}$, pois existe grande perda de eletrólitos e água livre para o terceiro espaço Estas alterações, frequentes na peritonite, pneumonia, diarreia, emese ou hipoadrenocorticismo, agravam ainda mais a condição inicial ${ }^{17}$, e também a fluidoterapia com solução de Ringer lactato de sódio utilizada neste estudo, pode ter contribuído para a iatrogenia ${ }^{17}$. Observou-se nos animais que vieram a óbito que a fração de excreção do sódio estava aumentada e de forma significativa $(\mathrm{p}<0,05)^{11,21}$.

A hipernatremia ${ }^{17}$ estava presente quando o proprietário abolia a dieta hídrica do seu animal para controlar a êmese e, em outros dois casos durante o tratamento com fluido, possivelmente por alterações iatrogênicas e desidratação. Nestes casos os animais receberam solução de cloreto de sódio a 0,45\%, para corrigir a desidratação ${ }^{17}$. Entretanto, na literatura a hipernatremia associada à desidratação era a alteração mais frequente, pois estava presente em 1/3 das cadelas com piometra ${ }^{7}$. Em contrapartida, foram encontrados casos isolados desta condição e com predomínio da hiponatremia, possivelmente pela gravidade e/ou maior tempo de evolução da doença nos animais por nós estudados.

Observou-se que os animais hipoclorêmicos (15/132) apresentavam-se com o bicarbonato próximo da normalidade, enquanto que 13 animais estavam hiperclorêmicos (13/132) e estavam com o bicarbonato abaixo dos valores de referência ${ }^{13,22}$. Ãsheim ${ }^{23}$, estudando a glomerulonefrite em cadelas com piometra, relacionou esta alteração com o espessamento da membrana basal dos túbulos renais ${ }^{23}$.

A hipocalemia foi detectada em $16 / 132$, no momento do diagnóstico e estas cadelas também apresentavam aumento do bicarbonato arterial, entretanto a hipercalemia esteve presente em oito casos (8/132) e geralmente acompanhado da diminuição do íon bicarbonato e do íon sódio. A hipocalemia é uma alteração frequentemente detectada nos casos de sepse por bactérias Gram negativas ${ }^{24}$, possivelmente decorrente do aumento na fração de excreção do potássio ${ }^{17}$ e também na alcalose respiratória que geralmente acompanha a bacteremia ${ }^{3}$.

Os animais estudados não apresentavam diferença quanto ao débito urinário (Tabela 4). As cadelas que, no segundo dia de tratamento, receberam uma dose maior de fluido em relação ao primeiro dia (Tabela 4) não sobreviveram ${ }^{11}$. Esta questão abre uma grande polêmica em relação à expansão volêmica com as doses maiores de fluido e risco de translocacão bacteriana ${ }^{18}$.

O exame de urina (Tabela 5) detectou alterações em relação à proteinúria, hemoglobinúria, possivelmente relacionada à hemólise. $\mathrm{O}$ pH urinário era ácido e reflete provavelmente o quadro de acidose metabólica ${ }^{10}$, alteração esta mais frequente antes de se iniciar o primeiro dia de tratamento. As mensurações dos eletrólitos na urina (Tabela 5) não se alteraram, mas exerceram grande impacto, quando foram calculadas as frações de excreção dos eletrólitos $(\mathrm{p}<0,05)$. De qualquer forma, a mensuração do eletrólito na urina do cão não reflete a fisiologia normal do rim, quando comparada ao homem ${ }^{25}$, pois vários animais normais foram acompanhados por Shaw ${ }^{26}$, que detectou grande concentração de eletrólitos na urina destes animais.

A URR na IRA foi padronizada em 25 a 33\% no cão nos primeiros três dias de diálise ${ }^{16}$ e ao analisar-se a URR dos animais sobreviventes e não sobreviventes, houve diferença significante $(\mathrm{p}<0,05)^{11}$, apesar de não ter sido detectada diferença durante o tratamen- 
to com fluido (Tabela 1). Valores negativos de URR estavam presentes nos animais que foram a óbito ${ }^{11} \mathrm{e}$ possivelmente a URR, obtida durante a fluidoterapia das cadelas com piometra, seja a mesma da diálise, pois os valores médios da URR estão muito próximos dos seus valores, ou seja, 25 a $33 \%$.

Monitorando diariamente o clearance de creatinina de animais com piometra (Tabela 2) e em síndrome urêmica, observou-se que 0,1 a $0,3 \mathrm{~mL} / \mathrm{kg} / \mathrm{min}$ correspondiam a índices graves de IRA ${ }^{11}$. Iniciou-se então a avaliação do tratamento com fluido com a mensuração do clearance da creatinina (Ccr); utilizou-se como resposta positiva ao tratamento conservador $\mathrm{Ccr}>$ $0,5 \mathrm{~mL} / \mathrm{kg} / \mathrm{mim}$. Entretanto, detectou-se que vários animais que apresentavam este índice também não se recuperavam da IRA em tratamento com fluido ou hemodiafiltração ${ }^{11}$. Após as experiências obtidas durante o estudo em tela acreditamos que o índice possivelmente sustentável para um animal com piometra e da IRA e após tratamento com fluido não deve exceder a limites inferiores de clearance, ou seja, $\mathrm{Ccr}<1$

\section{Referências}

1. SCHEPPER, J.; COCK, I.; CAPIAU, E. Urinary $\gamma$-glutamil transferase and the degree of renal dysfunction in 75 bitches with pyometra. Research inVeterinary Science, v. 46, p. 396400, 1989.

2. NELSON, R. W.; COUTO, C. G. Distúrbio da vagina e do útero. In: Medicina interna de pequenos animais. Rio de Janeiro: Guanabara Koogan, 1998. p. 681-684.

3. BORRENSEN, B. Pyometra in the dog. A pathophysiological investigation. VI Acid-base status and serum eletrolytes. Nordisk Veterinaermedicin, v. 36, p. 1-12, 1984.

4. BLAIR, E. Hypocapnia and Gram-negative bacteraemic shock. American Journal of Surgery, v. 119, p. 433-439, 1970

5. BEHREND, E. N.; GRAUER, G. F.; MANI, I.; GROMAN, R. P.; SALMAN, M. D.; GRECO, D. S. Hospital-acquired acute renal failure in dogs: 29 cases (1983-1992). Journal of the American Veterinary Medicine Association, v. 208, n. 4, p. 537-541, 1996.

6. STONE, E. A.; LITTMAN, M. P.; ROBERTSON, L. J.; BOVÉE, K. C. Renal dysfunction in dogs with pyometra. Journal of the American Veterinary Medicine Association, v. 193, n. 4, p. 457-464, 1988.

7. FRANSSON, B. A. Systemic inflamatory response in canine pyometra. The response to bacterial uterine infection. 2003. Thesis (Doctoral) - Swedish University of Agricultural Sciencis, Uppsala, 2003. p. 9-48.
$\mathrm{mL} / \mathrm{kg} / \mathrm{min}$., e nestes casos deveriam ser aplicadas as vantagens citadas na literatura de diálise precoce ${ }^{27}$, ou seja, diálise em indivíduos com clearance menor que $1 \mathrm{~mL} / \mathrm{kg} / \mathrm{min}$.

\section{Conclusões}

O tratamento com fluido na IRA da cadela com piometra com solução de Ringer lactato de sódio foi efetiva. A avaliação da responsividade à fluidoterapia pode ser mensurada pelo clearance da creatinina endógena e valores maiores ou iguais a $1 \mathrm{~mL} / \mathrm{kg} / \mathrm{min}$ são considerados como uma resposta positiva; valores menores que $1 \mathrm{~mL} / \mathrm{kg} / \mathrm{min}$ considerados resposta negativa e valores menores ou iguais a $0,5 \mathrm{~mL} / \mathrm{kg} / \mathrm{min}$ um preditor de falência renal grave e ou morte.

\section{Agradecimentos}

A FAPESP pelo apoio financeiro (Processo: 2004/03957-5); ao Dr Alexandre Libório pela assessoria estatística.

8. TOBIAS, K. M. S.; WHEATON, L. G. Surgical management of pyometra in dogs and cats. Seminars in Veterinary Medicine and Surgery (Small Animal), v. 10, n. 1, p. 30-34, 1995.

9. MUELLER, M. G.; LUDWIG, L. L. Use of closed-suction drains to treat generalized peritonitis in dogs and cats: 40 cases (1997-1999). Journal of the American Veterinary Medicine Association, v. 219, n. 6, p. 789-794, 2001.

10. COWGILL, L. D.; LANGSTON, C. E. Role of hemodialysis in the management of renal failure. The Veterinary Clinics of North America. Small Animall Practice. Renal Disfunction, v. 26, n. 6, p. 1347-1378, 1996.

11.FERREIRA, P. C. C. Avaliação da hemodiafiltração no período perioperatório da ovariossalpingohisterectomia, em cadelas com piometra e refratárias ao tratamento conservador da insuficiência renal aguda. 2006. $176 \mathrm{f}$. Tese (Doutorado) - Faculdade de Medicina Veterinária e Zootecnia, Universidade de São Paulo, São Paulo, 2006.

12.KUDNING, S. T.; MAMA, K. Guidelines for perioperative fluid therapy. Compendium, v. 25, n. 2, p. 102-111, 2003.

13. MUIR, W. W. Handbook of veterinary anesthesia. 3. ed. Ohio: Mosby, 2000. 574 p.

14.DELMAR, R. F. Evaluation of renal functions. In: OSBORNE, C. A.; FINCO, D. E. (Ed.). Canine and feline nephrology and urology. Philadelphia, Lippincott: Williams \& Wilkins, 1995. p. $216-229$. 
15.DAUGIRDAS, T. J.; BLAKE, P. G.; ING, T. S. Handbook of dialysis. 3. ed. Philadelphia, Lippincott: Williams \& Wilkins, 2001. p. 102-120.

16. ELLIOT, D. A. A hemodialysis. Clinical Techiniques in Small Animal Practice, v. 15, n. 3, p. 136-148, 2000.

17.DIBARTOLA, S. P. Fluid therapy in small animal practice. 2. ed. Philadelphia: Saunders Company, 2000.611 p.

18. AKAMINE, N.; FERNANDES, C. S. S. E.; BUCHELE, G. L.; PIVA, J. Hemodynamic support. Revista Brasileira de Terapia Intensiva, v. 16, n. 4, p. 241-245, 2004.

19.FALDYNA, M.; LAZNICKA, A.; TOMAN, M. Imunossupression in bitches with pyometra. Journal of Small Animal Practice, v. 42, n. 1, p. 5-10, 2000.

20.BRADY, C. A.; OTTO, C. M. Systemic inflamatory response syndrome, sepsis, and multiple organ dysfunction. Veterinary Clinics of North America: Small Animal Practice, v. 31, n. 6, p. 1147-1162, 2001.

21.MARTIN, L. G.; GROMAN, R. P. Relative adrenal insufficiency in critical ilness. Journal of Veterinary Emergency and Critical Care, v. 14, n. 3, p. 149-157, 2004.
22.GILL, J. R.; BARTLER, F. C. Evidence for a prostaglandinindependent defect in chloride reabsorption in the loop of Henle as a proximal cause of Bartter's syndrome. American Journal of the Medicine, v. 65, p. 766-772, 1978.

23. ÃSHEIM, Ã. Comparative pathophysyological aspects of the glomerulonephritis associated with pyometra in dogs. Acta Veterinary of Scandinávia, v. 5, p. 188-207, 1964.

24.KNOCHEL, J. P. The pathophysiology and clinical characterustics of severe hypophosphatemia. Archives of Internal Medicine, v. 137, p. 203-220, 1977.

25.HONSI, E. Insuficiência renal aguda em UTI. São Paulo: Atheneu, 1998. 304 p. (Clínicas Brasileiras de Medicina Intensiva).

26.SHAW, D. H. Relationship between urine ammonium ion excretion and d urine anion gap in dogs. American Journal of Veterinary Research, v. 52, n. 12, p. 1956-1959, 1991.

27.GETTINGS, L. G. Outcome in post-traumatic acute renal failure when continous renal replacement therapy is applied early vs. Late. Intensive Care Medicine, v. 25, p. 805-813, 1999. 